\title{
The influence of network topology on synchrony and oscillations in networks of spiking neurons
}

\author{
Duane Q Nykamp ${ }^{1 *}$, Alex Roxin ${ }^{2}$, Albert Compte ${ }^{2}$ \\ From Twentieth Annual Computational Neuroscience Meeting: CNS*2011 \\ Stockholm, Sweden. 23-28 July 2011
}

We use the recently developed framework of second order networks[1] to study the influence of network topology on synchrony and oscillations in networks of integrate-and-fire neurons. Second order networks are generalizations of Erdős-Rényi random networks [2], where one can specify the second order statistics among the network connections, i.e., the relative frequency of second order edge motifs that are embedded in the larger network. The second order edge motifs are the reciprocal, convergent, divergent, and chain connections diagrammed in Figure 1. Using the small number of parameters of the second order network framework, one can manipulate global network statistics such as the variance of the in- and out-degree distributions (through convergent and divergent connections) as well as the covariance between in- and out-degree (through chain connections).

Our previous work has identified motifs involving excitatory neurons that strongly influence the dynamical state of the network. In recurrent excitatory networks where the mean current caused neurons to fire, increasing chain connections led to higher network synchrony while increasing convergent connections decreased synchrony [1]. In networks of excitatory and inhibitory neurons where neuron firing was due to fluctuations of the membrane potential (the fluctuation-driven regime), broadening the excitatory incoming degree distribution onto excitatory neurons led to increased oscillations [3]. Since incoming degree distribution is tightly linked with convergent connections, this latter result demonstrated a strong effect of excitatory convergent connections on the network state. In both studies, the divergent connection motif (or the common input motif) did not have a large qualitative influence on the dynamics.

In the present study, we systematically investigate the influence of second order network motifs on the dynamical state of recurrent networks of excitatory and inhibitory neurons in the fluctuation-driven regime. We simulate networks of sparsely coupled integrate-and-fire neurons, where the connection probabilities are given by the second order network model. We allow the probability of each second order motif to depend on the populations (excitatory versus inhibitory) of the neurons involved in the motif. We again find that divergent connections alone have little qualitative effect on the dynamics. However, both convergent connections and

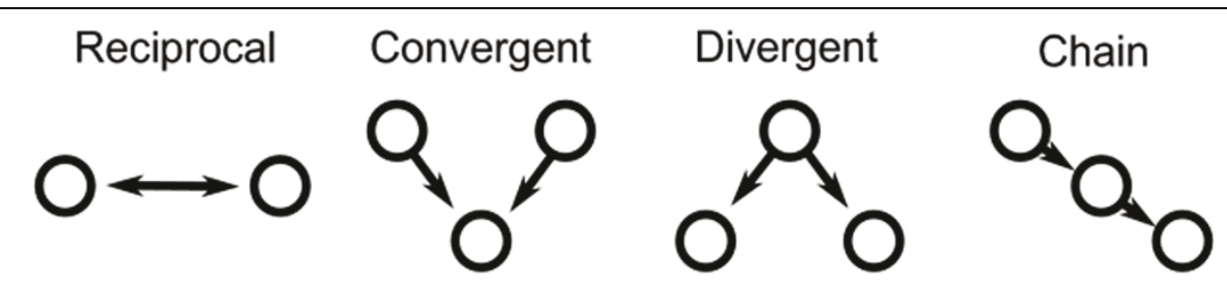

Figure 1 The four second order edge motifs of reciprocal, convergent, divergent, and causal connections.

\footnotetext{
* Correspondence: nykamp@umn.edu

'School of Mathematics, University of Minnesota, Minneapolis, MN 55455,

USA

Full list of author information is available at the end of the article
}

C Biomed Central

๑ 2011 Nykamp et al; licensee BioMed Central Ltd. This is an open access article distributed under the terms of the Creative Commons Attribution License (http://creativecommons.org/licenses/by/2.0), which permits unrestricted use, distribution, and reproduction in any medium, provided the original work is properly cited. 
chain connections dramatically affect the onset and magnitude of synchronous network oscillations, either increasing or decreasing oscillations depending on the populations of the neurons in the motif. We explain these results through analysis of a mean-field model of the coupled populations.

\section{Author details}

${ }^{1}$ School of Mathematics, University of Minnesota, Minneapolis, MN 55455, USA. ${ }^{2}$ Institut d'Investigacions Biomèdiques August Pi i Sunyer (IDIBAPS),

Barcelona, Spain.

Published: 18 July 2011

\section{References}

1. Zhao L, Beverlin B, Netoff T, Nykamp DQ: Synchronization from second order network connectivity statistics., Submitted.

2. Erdős P, Rényi A: On Random Graphs. I. Publicationes Mathematicae 1959, 6:290-297.

3. Roxin $\mathrm{A}$ : The role of degree distribution in shaping the dynamics in networks of sparsely connected spiking neurons. Front. Comput. Neurosci 2011, To appear.

doi:10.1186/1471-2202-12-S1-P44

Cite this article as: Nykamp et al:: The influence of network topology on synchrony and oscillations in networks of spiking neurons. BMC

Neuroscience 2011 12(Suppl 1):P44.

\section{Submit your next manuscript to BioMed Central} and take full advantage of:

- Convenient online submission

- Thorough peer review

- No space constraints or color figure charges

- Immediate publication on acceptance

- Inclusion in PubMed, CAS, Scopus and Google Scholar

- Research which is freely available for redistribution

Submit your manuscript at www.biomedcentral.com/submit 